\title{
A DESCRIPTION OF HAEMOGREGARINA SPECIES NATURALLY INFECTING WHITE-SPOTTED GECKO (TARENTOLA ANNULARIS) IN QENA, EGYPT \\ By
}

\author{
SOHEIR A.H. RABIE AND ABDEL-NASSER A. HUSSEIN \\ Department of Zoology, Faculty of Science, South Valley University, Qena, Egypt \\ Abstract
}

The present study describes the developmental stages of Haemogregarina species in the blood and tissues of naturally infecting white-spotted gecko Tarentola annularis collected from Qena, Egypt. Different parasite's forms were observed infecting the erythrocytes. The gamonts enclosed within parasitophorous vacuole and seems to have no clear effect on the host cell especially in case of immature parasite forms. But in the presence of mature gamonts the host cell nucleus displaced. The parasitaemia level is up to 280 per 10,000 erythrocytes counted. Trophozoites and gamonts have been recognized in the blood smears. The rounded trophozoite diameter is $3.84 \pm 0.87 \mu \mathrm{m}$, while the elongated trophozoite measured $4.42 \pm 0.69 \times 2.8 \pm 0.56 \mu \mathrm{m}$. The mature gamonts were differentiated into two forms; short gamont measuring $10.82 \pm 0.82 \times 3.30 \pm 0.73 \mu \mathrm{m}$ (range: $10-12.1 \times 2.2-4.4 \mu \mathrm{m})$ and the long gamont measured $14.67 \pm 0.83 \times 3.96 \pm 0.77 \mu \mathrm{m}$ (range: $14.1-16.5 \times 3.3-5.5 \mu \mathrm{m}$ ). Merogony carried out only in the endothelial cells of the blood capillaries in the lung. Different merogonic stages have been recognized and differentiated in two forms; micromeront measured $13.25 \pm 0.50 \times 12 \pm 0.0 \mu \mathrm{m}$ and produces a few number of large merozoites, macromeront measured $19.75 \pm 0.87 \times 13.25 \pm 0.50 \mu \mathrm{m}$ and produces more small sized merozoites. The gamonts and merozoites have the general characteristic ultrastructures of the Apicomplexa containing components of the apical complex, e.g. pellicle, micronemes, rhoptries, and few dense bodies and subpellicular microtubules.

Key words: Haemogregarina, Tarentola annularis, Parasitaemia, Light and Electron microscope, Egypt.

\section{Introduction}

Reptiles are very old in the evolutionary scale and their parasites seem to become stabilized allover the world. Parasitism causes deleterious effects on several aspects of the ecology and evolution of hosts (Shazly 2000; Smallridge and Bull, 2000; Eisen, 2001; Amo et al, 2005).

Plimmer (1912) reported haemogregarines in Tarentola (T.) annularis and Ptyodactylus (P.) lobatus from Egypt. Several records of haemogregarines from reptiles in various parts of the world are known (Chatton and Blanc 1914; Foley and Catanei 1925; Saoud and Younis 1969; El-Wasila, 1989; Shazly 2000; Amo et al, 2004; 2005; Bouma et al, 2007; Al-Farraj 2008; Mihalca et al, 2008; Cook et al, 2009; Abdel-Baki and AlQuraishy, 2012; El-Toukhy et al, 2013; Abdel-Haleem et al, 2013).
Haemogregarines infecting $T$. annularis were studied by many authors (El-Naffar et al, 1991; Ramadan et al,1995; Saoud et al,1995; Mohammed and Ramadan, 1996; Rabie et al, 2006; Abd-Al Aal et al, 2009). Haemogregarina species infecting the geckoes $P$. hasselquistii were also studied (Abdel-Ghaffar et al, 1994; Saoud et al, 1995; Hussein, 2006). Martínez-de La Puente and Merino (2008) supported the hypotheses proposed that the multiple invasions being due to higher parasite densities. The parasitaemia level of haemogregarines infecting Mabuya (M.) quinquetaeniata was $1 \%-8 \%$ and in some cases $40 \%-50 \%$ of the erythrocytes (Bashtar et al, 1987). While in $P$. hasselquistii infected with haemogregarine (Karyolysus) was up to 410/10,000 counted erythrocytes (Hussein, 2006). The parasitaemia level was 200-400/1000 erythrocytes of haemogregarines infecting 
Cerastes c. gasperitti (Al-Farraj, 2008). In M. quinquetaeniata parasitized by Hepatozoon gracilis the level was slight $(\leq 2 \%)$, moderate (3-10\%) and high (11-20\%) per 1000 counted erythrocytes (Galal, 2010). Electron microscopy was used in studying blood and merogonic stages of haemogregarines in reptiles (Abdel-Ghaffar et al, 1994; Desser 1997; Smallridge and Paperna, 2000; Jovani et al, 2004; Rabie et al, 2006; Hussein, 2006).

The present study aimed to renew knowledge on the blood and tissue stages of haemogregarines infecting the Egyptian gecko, T. annularis.

\section{Materials and Methods}

Geckoes were handy captured and maintained separately in cages with suitable food and enough water at room temperature (28$35^{\circ} \mathrm{C}$ ). Blood films were made, stained in Giemsa and examined by light microscope. From the highly infected animals, small pieces $(2 \mathrm{~mm})$ of lung and liver were washed in $0.9 \%$ physiological saline solution, fixed in $3 \%$ glutaraldehyde buffered in $0.1 \mathrm{M}$ sodium cacodylate $(\mathrm{pH} 7.3)$, then washed in the same buffer, postfixed in $2 \%$ osmium tetroxide, washed in the buffer and dehydrated in ethanol. The specimens were embedded in araldite. Semithin sections were made on a Reichert microtome (thickness $0.5-0.7 \mu \mathrm{m})$, stained using methylene blue and examined by a Leica research photomicroscope. Ultrathin sections $(40-50 \mathrm{~nm})$ were made and stained and contrasted using lead citrate and uranyl acetate (Reynolds, 1963) and then examined with a JEOL transmission electron microscope (JEM1010). The blood and tissue stages (from the semithin section) were observed and photographed using a Leica DM1000 microscope with a Leica EC3 camera. Measurements were done with an eyepiece micrometer with an oil-immersion lens, and given in micrometers as: mean \pm standard deviation followed by the range in parentheses. The present data have been compared with those previously described (Tab. 1).

\section{Results}

Blood stages and parasitaemia: In blood smears of infected $T$. annularis, different forms of the blood stages were observed infecting the erythrocytes and none of the leucocytes are infected. The parasites are usually intracellular. The infected erythrocytes were not affected by the presence of parasites. The immature blood stages of parasite have no effect on the host cell nucleus, but in case of infection with mature gamont the host cell nucleus had been displaced. Also the parasites have no karyolytic effect (Figs. 1\&2). The parasitophorous vacuole surrounding blood and tissue stages were recognized using light microscope around some parasite stages (Fig. 3). Only single gamont and very rarely double gamonts were observed in the infected erythrocyte (Fig. 4). The gamont did not attack leucocytes. No fin-folds were seen within the gamont. The parasitaemia level (number of parasite/10.000 erythrocytes) was up to 280/10,000 erythrocytes. Host cell seemed to be not highly affected, where the infected cells measured $15.18 \pm 1.78 \times 10.56 \pm 0.77 \mu \mathrm{m}$ (range: $13.2-17.6 \times 9.9-11.0 \mu \mathrm{m}$ ) and the uninfected one measured $16.80 \pm 1.53 \times 10.12 \pm$ $0.70 \mu \mathrm{m}$ (range: $14.0-18.7 \times 9.9-8.8 \mu \mathrm{m}$ ) in length and width, respectively.

In blood smears, trophozoites and gamonts were recognized. The trophozoites appears firstly rounded and then elongated (Figs. $1 \& 2)$. The rounded form diameter measured $3.84 \pm 0.87 \mu \mathrm{m}$ (range: $3-4.4 \mu \mathrm{m}$ ), while the elongated trophozoite measured $4.42 \pm 0.69 \times$ $2.8 \pm 0.56 \mu \mathrm{m}$ (range: $3.3-5 \times 2.2-3 \mu \mathrm{m}$. The mature gamonts (Figs. $2 \& 3$ ) were differentiated into two forms; short gamont measuring $10.82 \pm 0.82 \times 3.30 \pm 0.73 \mu \mathrm{m}$ (range: $10-12.1 \times$ 2.2-4.4 $\mu \mathrm{m}$ ) and the long gamont measured $14.67 \pm 0.83 \times 3.96 \pm 0.77 \mu \mathrm{m}$ (range: $14.1-16.5$ $\times 3.3-5.5 \mu \mathrm{m})$.

Merogony and merozoites: Meronts in different developmental phases were seen in few numbers - in the endothelial cells of the blood capillaries of the lung (Figs. 5-8). 
But, none was seen in parenchyma cells of infected geckos' liver.

Merogony begins by the invasion of the parasite (sporozoite) to the endothelial cells of the blood vessels in the lung. The parasite became more rounded and began to grow in size forming developing meronts. The parasitophorous vacuole appeared enclosing the parasite and grows in size during the growth of the meront. The formation of merozoites began with the division of parasite nucleus into nuclei. Few daughter nuclei were seen peripherally arranged in the meront (Figs. 57). The cytoplasm remained, after the separation of the merozoites, appeared as a residual body (Fig. 7). The nuclei of the multinucleated meront undergo elongation and finally formed merozoites (Figs. 7\&8). There were different merogonic stages; mononucleated meront (Fig. 5) and polynucleated meront (Figs. 6-8). According the measurements, the meronts are present in two forms; the small form (micromeront) measured $13.25 \pm 0.50 \times 12 \pm 0.0 \mu \mathrm{m}$ and produces a few number of large merozoites (Figs. 5\&6). The large form (macromeront) measured $19.75 \pm 0.87 \times 13.25 \pm 0.50 \mu \mathrm{m}$ and produces more small sized merozoites (Figs. 7\&8).

By the ultrastructure study, the gamonts and merozoites have the general characteristic ultrastructures of the Apicomplexa; containing components of the apical complex, e.g. pellicle, micronemes, rhoptries, very few dense bodies and subpellicular microtubules, which were located mainly at one pole of the parasite. Also residual body had seen after the merozoites formation (Fig. 9).

\section{Discussion}

Haemogregarines are apicomplexan protozoa belong to a group of blood protozoa, in which gamonts infected blood cells of reptiles and some other vertebrates. Merogony takes place in certain internal organs of the vertebrate hosts whilst sporogony occurs in invertebrate hosts (Levine 1982). Classification of haemogregarines depends on many criteria as site of schizogony and mode of transmission after the specific sporogonic process within its haematophagus vector as well as its host range (Bashtar et al, 1984; Ahmed 1998; Shazly, 2000).

In present study, no marked effect was induced by the parasite to the infected erythrocyte. Also the host cell nucleus didn't affected neither in position nor its shape or structure due to immature parasite stages occurrence, but the host cell nucleus has displaced in case of presence of mature gamonts. This result partially disagreed with Saoud et al. (1995), where trophozoite and gametocyte stages of $H$. helmymohammedi caused the erythrocytes to be hypertrophied, stretched and stain paler than the uninfected ones. Also, the host cell nucleus usually displaced by parasites, and also, disagreed with the study carried out by Al-Farraj (2008) where infected erythrocytes were hypertrophied and elongated. Hussein (2006) and Abdel-Haleem (2013) reported that the infected erythrocytes were hypertrophied with their nuclei either longitudinally stretched or split into two fragments. The present data agreed with Saoud et al. (1995) in case of $H$. rawashi Mohammed and Ramadan 1996 on only displacment the host cell nucleus by gamonts, but disagreed in case of immature gamont presence.

Regarding intensity of infection, nearly all studies included the parasitaemia level, but very few discussed its importance. About the effect of parasitaemia of Hepatozoon gracilis infecting M. quinquetaeniata on some biological aspects of vertebrate and invertebrate hosts, Galal (2010) observed that the daily mortality rate increased gradually with the increase in parasitaemia level. Also, Hussein (2006) reported the parasitaemia level in case of Karyolysus sp. infected $P$. hasselquisti, but didn't refer to parasitaemia effect on hosts. Bashtar et al. (1987) considered the parasitaemia (1\%-8\%) in skinks was high and in some cases $40 \%$ $50 \%$ of the erythrocytes were parasitized. The present study reported a moderate level of parasitaemia $(280 / 10.000)$. 
Abdel-Ghaffar et al. (1994) differentiated the gamonts of Haemogregarina sp. infecting $P$. hasselquistti into two forms; small meront measured $10 \times 5 \mu \mathrm{m}$, while and the second form was large, measured $24 \times 9 \mu \mathrm{m}$ and represented the mature gamont. Saoud et el. (1995) described H. helmymohammedi as new species infecting Hemidactylus flaviviridis and redescribed $H$. rawashi Mohammed and Ramadan, 1996 infecting P. hasselquisti and $H$. tarentanulari parasitizing $T$. annularia. These data show differences with the data of the present materials. Rabie et al. (2006) described a haemogregarine species from $P$. hasslquisti, which had two forms of blood stages; short measured $8 \times 4 \mu \mathrm{m}$ and the large gamont measured $13 \times 4 \mu \mathrm{m}$. The merogont occurred in the host's lung and liver. The merogony stages were differentiated into two forms; micromeront measured $12 \times 9 \mu \mathrm{m}$ and macromeront $20 \times 13 \mu \mathrm{m}$. The present forms of the gamonts and meronts were different by measurements and merogony was found only in lung.

The present gamonts were seemly close to the gamonts of haemogregarine species (Karyolysus) described by Hussein (2006), but infecting different host, $P$. hasslquisti, and also the previously parasite had a karyolytic effect on the host cell nucleus. The present gamonts have no karyolytic effect on the host cell nucleus. Comparison of host, geographical distribution and measurements between the present species and the previously described Haemogregarina species in geckoes from different localities in Egypt revealed that the present Haemogregarina species infection T. annularis was not identical to any of those previously described.

Regarding the merogony in the previously mentioned parasites were found in lung and liver. Merogony was observed only in the endothelial cells of the blood capillaries in the lung only of $P$. hasselquistti (AbdelGhaffar et al, 1994). In the present study, merogony was found only in lung and the different measurements of the present tissue stages differed from those previously de- scribed. The cyst forming coccidians maintained their 3-layered pellicle during schizogony (Abdel-Ghaffar et al, 1994). The schizont's nucleus eventually underwent repeated divisions and the resulting daughter nuclei migrated to the periphery of the schizont directly underneath the outer boundary. The first indication of merozoite formation was a thickening and protrusion of the schizont boundary at sites where a nucleus was close to this membrane and this structure is termed merozoite anlage. Similar observations were reported on the coccidia as well as the subsequent development and formation of merozoites, where such process was described as ectomerogony (Bashtar et al, 1984; Shazly, 2000). The fine structure of the merozoite was similar to that described for the erythrocytic stages of the parasite, except for the presence of more amylopectin granules in the merozoites. This was observed in many coccidian merozoites (Bashtar et al, 1984; Ahmed, 1998; Shazly, 2000; Mehlhorn, 2001). The cell boundary of the erythrocytic gamonts and meronts are consisted of an outer unit membrane and an inner complex of 2 closely opposed unit membranes (Abdel-Ghaffar et al, 1994; Hussein, 2006). In the present material, the ultrastructure showed that each parasite was covered by a pellicle consisting of two layers, (but not easy to recognize if the inner membrane complex seen to resolve into two unit membranes). The present study showed that the gamont had the structural elements of the apicomplexa as the two membranes pellicle, subpellicular microtubules, rhoptries, homogenously distributed micronemes and some of dense bodies were distributed in the cytoplasm. Also the daughter nuclei had been peripherally arranged.

\section{References}

Abd-Al Aal, Z, Aly, E, Ahmed, S, El-Raey, S, 2009: Studies on haemogregarine species parasite of white spotted gecko (Tarentola annularis). The $4^{\text {th }}$ Environmental Conference, Faculty of Science, Zagazig University, Egypt.

Abdel-Baki, AS, Al-Quraishy, S, 2012: Morphological characteristics of a new species of 
Haemogregarina Danilewsky, 1885 (Apicomplexa: Adeleorina) in naturally infected Acanthodactylus boskianus (Daudin) (Sauria: Lacertidae) in Egypt. Sys. Parasitol. 82:65-9.

Abdel-Ghaffar, F, Abdel Aziz, A, El-Toukhy, A, Abdel-Gawad, M, 1994: Light and electron microscopic studies on the blood stages and merogony of Haemogregarina sp. infecting the gecko, Ptyodactylus hasselquistii. J. Egypt. Ger. Soc. Zool. 14, D:341-63.

Abdel-Haleem, HM, Al-Qurashy, S, AbdelBaki, AS, 2013: A redescriptian of Haemogregarina damiettae Ramadan et al. 1996 naturally infecting the Acanthodactylus boskianus from Egypt, with new merognic data. Parasitol. Res. 112:2045-8.

Ahmed, AK, 1998: Light and electron microscope studies of Hepatozoon Mehlhorni, Bashtar et al, 1991 (Adeleina, Eucoccidiida) naturally infecting the Viper Echis carinatus. J. Egypt. Soc. Parasitol. 28, 3:883-97.

Al-Farraj, S, 2008: Light and electron microscopic study on a haemogregarine species infecting the viper Cerastes cerastes gasperitti from Saudi Arabia. Pak. J. Biol. Sci. 11:1414-21.

Amo, L, López, P, Martín, J, 2004: Prevalence and intensity of haemogregarinid blood parasites in a population of the Iberian rock lizard, Lacerta monticola. Parasitol. Res. 94:290-3.

Amo, L, López, P, Martín, J, 2005: Prevalence and intensity of haemogregarine blood parasites and their mite vectors in the common wall lizard, Podarcis muralis. Parasitol. Res. 96:378-81.

Bashtar, AR, Abdel-Ghaffar, FA, Shazly, M A,1987: Developmental stages of Hepatozoon gracilis (Wenyon, 1909) comb. nov., a parasite of the Egyptian skink, Mabuya quinquetaeniata. Parasitol. Res. 73:507-14.

Bashtar, AR, Boulose, R, Mehlhorn, H, 1984: Hepatozoon aegypti nov.sp. 1- Life cycle. Z. Parasitenkd. 70:29-41.

Bouma, MJ, Smallridge, CJ, Bull, CM, Komdeur, J, 2007: Susceptibility to infection by a haemogregarine parasite and the impact of infection in the Australian sleepy lizard Tiliqua rugosa. Parasitol. Res. 100: 949-54.

Chatton, E, Blanc, G, 1914: Sur une hematozaire nouvaeu, Pirhemocyton tarentola, du geck, Tarentola mauritanica, et sur les alterations globularis qu'il determine. C. R. Soc. Biol. 77: 496-8.

Cook, CA, Smit, NJ, Davies, AJ, 2009: A redescription of Haemogregarina fitzsimonsi Dias,
1953 and some comments on Haemogregarina parvula Dias, 1953 (Adeleorina: Haemogregarinidae) from southern African tortoises (Cryptodira: Testudinidae), with new host data and distribution records. Fol. Parasitol. 56:173-9.

Desser, SS, 1997: Blood parasites of the iguanid lizard, Ctenosaura similis from Costa Rica, with a description of Hepatozoon gamezi n. sp. J. Eukaryot. Microbiol. 44:162-7.

Eisen, RJ, 2001: Absence of measurable malaria-induced mortality in western fence lizards (Sceloporus occidentalis) in nature: a 4-year study of annual and over-winter mortality. Oecologia 127:586-9.

EI-Naffar, MK, Mandour AM, Mohammed, MBH, 1991: Haemogregarina annularis n. sp. from the gecko Tarentola annularis in Assiut governorate, A. R. Egypt Bull. Fac. Sci. Assiut Uni. 20:217-26.

El-Toukhy, AA, Abdel-Aziz, A, Abo-Senna, FM, Abou El-Nour, MF, 2013: Three coccidian parasites from Moorish gecko, Tarentola mauritanica (Gekkonidae) 2- Eimeria alexandriensis n. sp. (Apicomplexa: Eimeriidae). Int. J. Adv. Res. 1, 7:526-34.

El-Wasila, M, 1989: Haemogregarina sp. (Apicomplexa: Adeleorina) from the gecko $\mathrm{Ta}$ rentola annularis in the Sudan: fine structure and life-cycle trials. Parasitol. Res. 75: 444-8.

Foley, H, Catanei, A, 1925: Haemogregarines de saurian d'Algerie, Arch. Inst. Past. Algerie 3:344-51.

Galal, FH, 2010: Effect of the intensity of Hepatozoon gracilis parasitaemia on some biological aspects of Culex (Culex) pipiens (Diptera: Culicidae). Egypt. Acad. J. Boil. Sci. 3, 1:57-66.

Hussein, ANA, 2006: Light and transmission electron microscopic studies of a haemogregarine in naturally infected fan-footed gecko (Ptyodactylus hasselquistii). Parasitol. Res. 98: 468-71.

Jovani, R, Amo, L, Arriero, E, Krone, O, Marzal, A, et al, 2004: Double gametocyte infections in apicomplexan parasites of birds and reptiles. Parasitol. Res. 94: 155-7.

Levine, ND, 1982: Some correction in haemogregarine (Apicomplexa: Protozoa) nomenclature. J. Protozool. 29: 601-603.

Martínez-de la Puente J, Merino, S, 2008: Mini-Review: Factors Affecting Multiple Invasions of Erythrocytes in Plasmodium and other 
Malaria-like Parasites: A neglected characteristic of infections. Open Parasitol. J. 2:40-2.

Mehlhorn, H, 2001: Encyclopedic References of Parasitology, $2^{\text {nd }}$ ed., Springer, Berlin, Germany.

Mihalca, AD, Racka, K, Gherman, C, Ionescu, DT, 2008: Prevalence and intensity of blood apicomplexan infections in reptiles from Romania. Parasitol. Res. 102, 5:1081-3.

Mohammed AHH, Ramadan NF, 1996: Description of two new haemogregarines from Egyptian geckos. J. Fac. Vet. Med. Cairo Uni. 3: 244-50

Plimmer, HG, 1912: On the blood parasites found in animals in the Zoological Garden during four years 1908-1911. Proc. Zool. Soc. London 82, 2:406-19.

Rabie, SM, Hussein, ANA, Schaub GA, 2006: Light and transmission electron microscope studies of a haemogregarine in naturally infecting Egyptian gecko (Tarentola annularis). J. Egypt. Ger. Soc. Zool. 94, D:23-37.

Ramadan, NF, Mohammed SH, Fawzi S M,1995: The Ultrastructure of some stages of Haemogregarina tarentannulari infecting the gecko Tarentola annularis. Qatar Uni. Sci. J. 15. 2:379-87.

\section{List of Figures}

Figs. 1-4: Light micrographs of Giemsa stained blood smears of Haemogregarina species naturally infecting gecko Tarentola annularis: Fig. 1: Rounded trophozoite (RT) with not affected host cell nucleus (HCN); Fig. 2 Elongated trophozoite (ET) with not affected host cell nucleus (HCN), also showing long gamont (LG) and slightly displaced host cell nucleus (HCN); Fig. 3: Short gamont (SG) surrounded by parasitophorus vacuole (PV), and displaced host cell nucleus (arrow head), also showing long gamont (LG) and displace host cell nucleus (arrow head); Fig. 4: Double infected erythrocyte by long gamont (LG) without affection on host cell or its nucleus $(\mathrm{HCN})$.

Figs. 5-8: Light micrographs of semi-thin sections in lung of T. annularis infected with Haemogregarina species: Fig. 5: A micromeront (MI) containing one daughter nuclei (DN); Fig. 6: Micromeront (MI) possesses four daughter nuclei (DN); Fig. 7: A macromeront (MA) contains peripherally arranged developing merozoites (M), and show residuale body (RB); Fig. 8: A macromeront (MA) contains developing merozoites (M).

Fig. 9: Transmission electron micrograph of macromeront in lung of T. annularis infected with Haemogregarina species, showing a macromeront (MA) surrounded by parasitophorus vacuole (PV) in lung endothelial cells containing developing micromerozoites $(\mathrm{M})$ and amylopectin granules $(A G)$ and residual body (RB). 


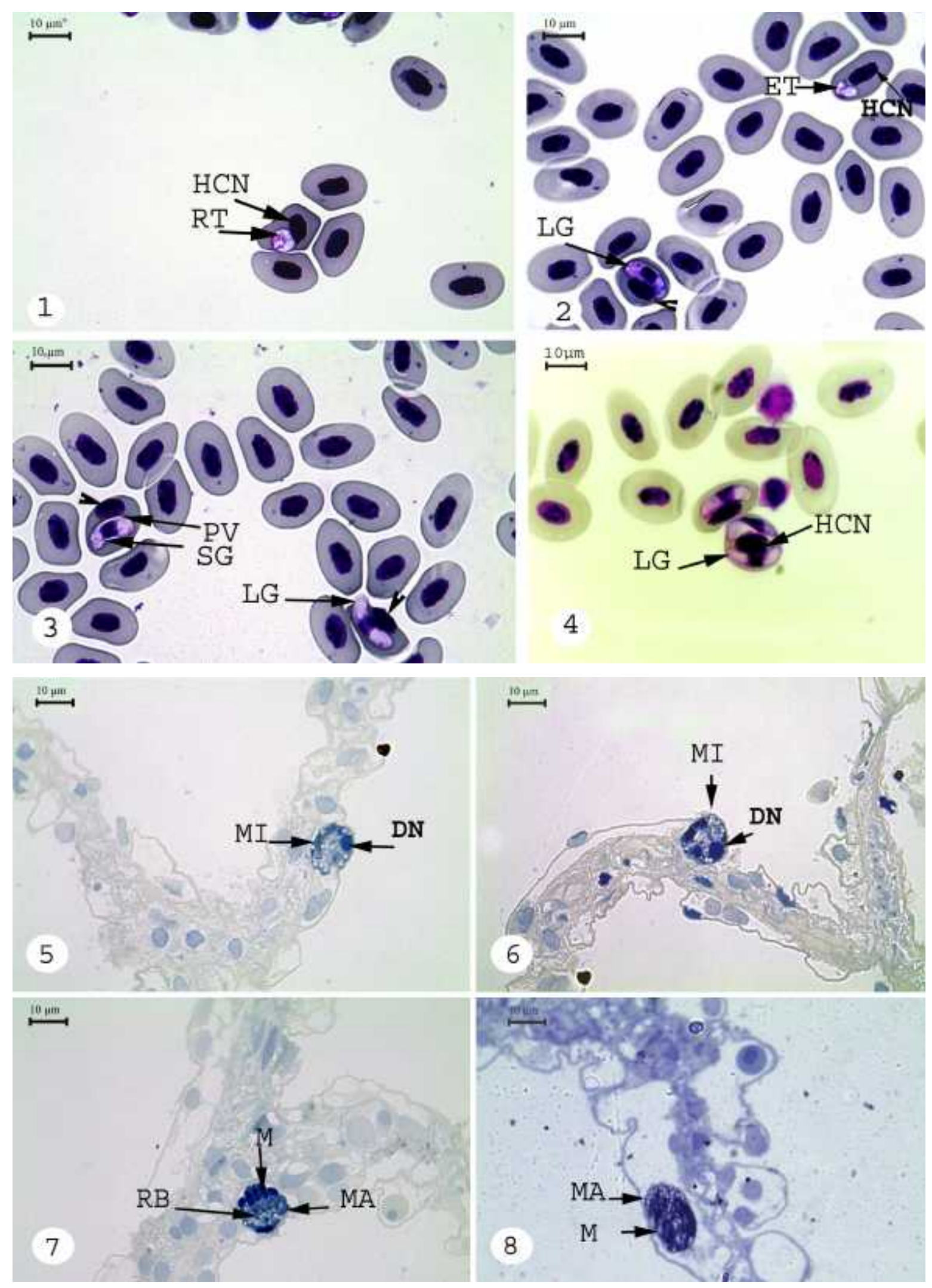




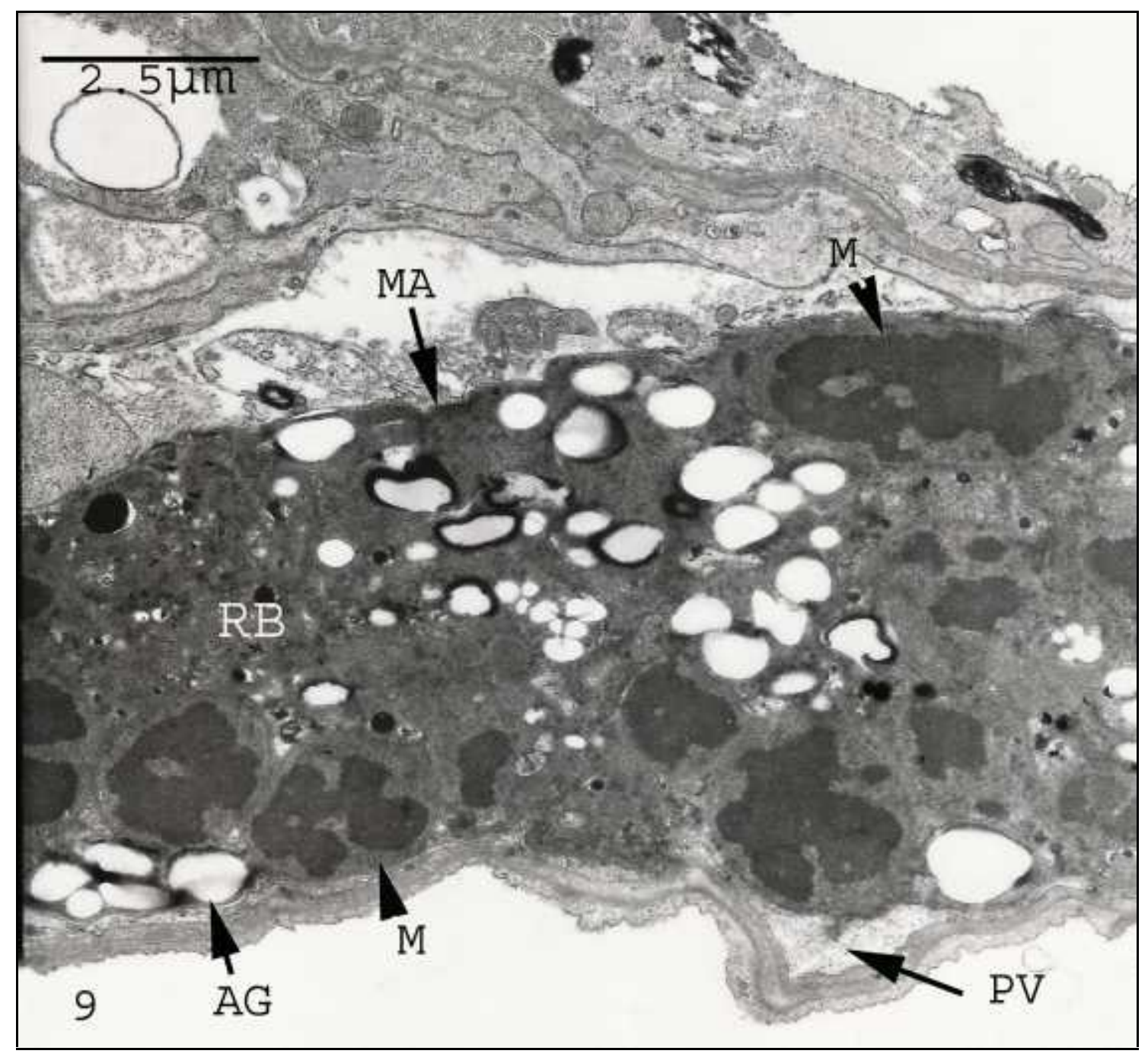




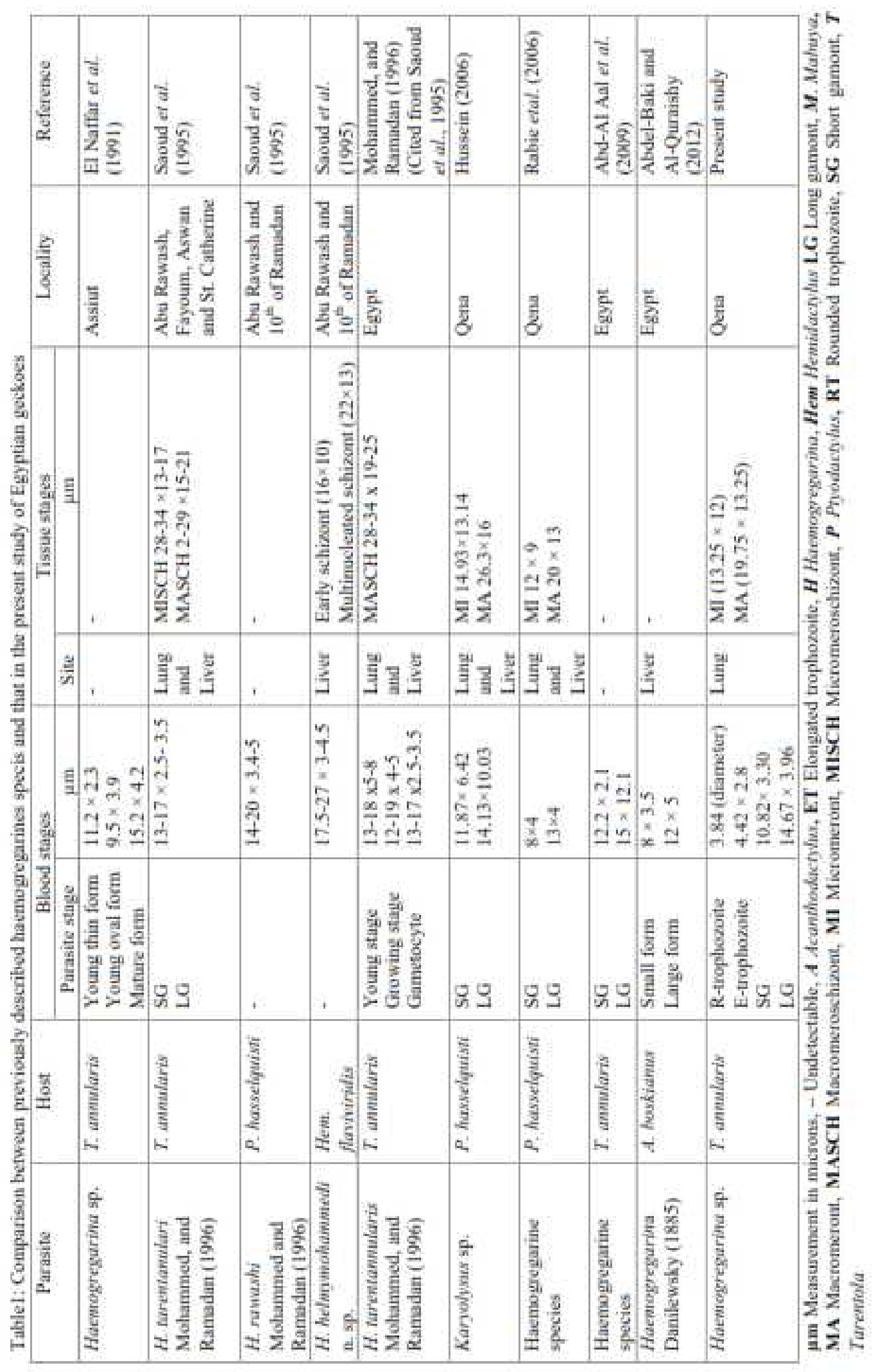

